\title{
A Study of Intelligent Hospital Environment Monitoring Method Based on the Android Platform
}

\author{
Wen-Cheng Cui, Hong Ren, Yang-Yang Han, Hong Shao \\ School of Information Science and Engineering \\ Shenyang University of Technology \\ Shenyang, 110870, China \\ shaoh1974@hotmail.com
}

\begin{abstract}
With the development of the medical treatment and public health field, the higher quality of hospital environment is required. This paper presents an intelligent hospital environment monitoring system based on the Android platform to overcome the present monitoring problems, such as incomprehensive monitoring data, delayed alert, lower intelligence of level and etc. This new method introduced will greatly reduce the waste of hospital human resource, improve the service level of the hospital and make a big contribution to further realizing the safe city.
\end{abstract}

Keywords-hospital environment monitoring; android; safe city; WIFI ;intelligent alarm

\section{INTRODUCTION}

Hospital is always regarded as a protective umbrella for people's health. The environment in it directly affected the recovery of patients. The national air quality standard, which is carried in 2002, definitely set the standards for the environment parameters, such as temperature, humidity, noise, gas, inhalable particle, VOC and bacterial total [1]. In 2007, Beijing introduced the hospital infectious disease department internal air quality requirements, in which, the standards of environment parameters are listed in detailed [2]. Moreover, it has been carried out with a strong hand. But for the domestic hospital monitoring, now is still in an artificial periodic period. It remains a lot of inadequacy both in realtime and intelligent information.

According to the report from Canalys, which is a market research company, in 2011, it is the first time that the Smartphone won the PC including Tablet PC in global sales [3]. Android mobile platform based on the Linux core, is quickly taking a large share of the global market with its open and intelligent features. In this paper, a system based on Android, combining Android Service and multithread technology, is designed for the hospital environment monitoring. This system can remotely control and get realtime environment information, which definitely can solve previous environment monitoring problems.

\section{GENERAL FRAMEWORK DESIGN}

In this paper, an intelligent hospital environment monitoring system is designed on the Android platform due to the problems in present hospital mornitoring system, such as monitoring not in time, alarming not intelligent, large waste of resources and so on. The overall framework consists of three parts: the lower computer, the server and the Android platform, which is shown in the Fig. 1. By the multiple sensors, the lower computer collects the data of temperature, humidity, light, $\mathrm{CO} 2$, noise and dust particles, and then the datas are transmited to the server on WIFI.The server part is mainly responsible for analyzing and storing the data by using a double buffer memory, which provides a data accessing interface for Android platform accessing these data. The Android platform, as the core in this design, is mainly divided into four modules: data transceiver module, data analysis module, background monitoring module, and foreground display module. The communication between four modules is realized by Android multi-threaded technology.

The working process of Android platform is as follows: Firstly, the foreground monitoring triggers user event, and then sends command to data transceiver module. Data transceiver module is responsible for communication with the interface of sever. Data returned by the server is committed to the data analysis module through data transceiver module. Then these data which have been analyzed by data analysis module, real-time display in foreground display module through thread intercommunications. Intelligent alarm is handled by background monitoring module. Similarly, data transceiver module is responsible for sending and receiving data, data analysis module is responsible for parsing data. If the analysis result doesn't meet standard, the system will announce alarm signal and display signal through foreground module. Finally, hospital achieves mobile intelligent monitoring of the environment information.

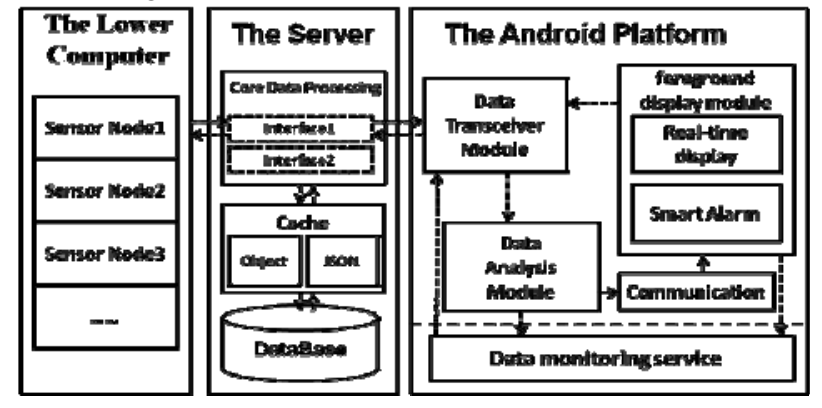

Figure 1. The General Framework 


\section{DATA TRANSMISSION AND ANALYSIS METHOD}

The android application can be connected to the network via GPRS, WIFI and so on. GPRS is a mobile network service provided by the mobile operators. This business primarily use the SIM card on this network for sending and receiving signals between the signal receiving tower and your mobile phone. WIFI is a wireless network composed of the AP (Access Point) and wireless network card. The wireless network card is responsible for receiving the signals emitted by the AP client terminal devices, and the android applications manage and apply WIFI by obtaining access to WIFI. By either way, the communication with the server will be established when the client is connected to the network by using the HTTP protocal. This paper presents a communication protocol consists of two parts: the HTTP protocal in bottom layer and the self-defining communication protocol in the upper layer. The format of the upper layer communication protocol is realized by JSON which is a lightweight data interchange format and also easier to read and write [4]. The whole transfer process is as follows:

First, establish a connection at the terminal of the data transmission module in Android application. Create a HttpURLConnection object and send the parameters to the server via GET or POST method.

Second, the required environmental data will be assembled and sent to the Android terminal as JOSN format in the server.

Last, the data analysis module analysis the JSON data stream in the android terminal, and the Specific analytic process is shown in Figure 2.

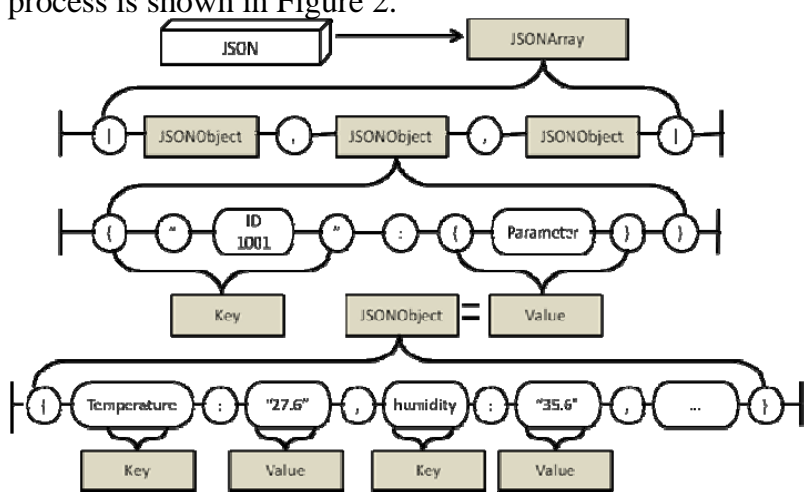

Figure 2. JSON Data Analytic Process

\section{DATA MONITORING SERVICE IN BACKGROUND}

After Connecting with server, the environment monitoring method can be executed. In order to enhance the experience of user, we select background service from the monitoring methods; this design can guarantee the environment monitor when user begins some other operation. Specific method is shown in Fig. 3.

First, start the background service promptly when the user presses the service button, and meanwhile register broadcast receiver for monitoring the illegal environment information and then update UI. When the background service is open, another broadcast will be registered for monitoring the end service event, and at the same time open a new thread to communicate with server. The server will send the environment information of the department regularly based on the authority of the user. After passing throw the transceiver module and analysis module, the data will arrive at the alarm strategy module. The system will give a feedback of the alarm strategy to user by analysis the environment data through data fusion technology. During operating the system, some other user events will not influence the monitoring service when the background service in the open state. The service will not stop until the user trigger the stop service button. It is noteworthy that multithread must be needed when the background service designed. Besides it needs stop both the service thread and monitoring service in foreground Activity in order to obtain information form the server regularly.

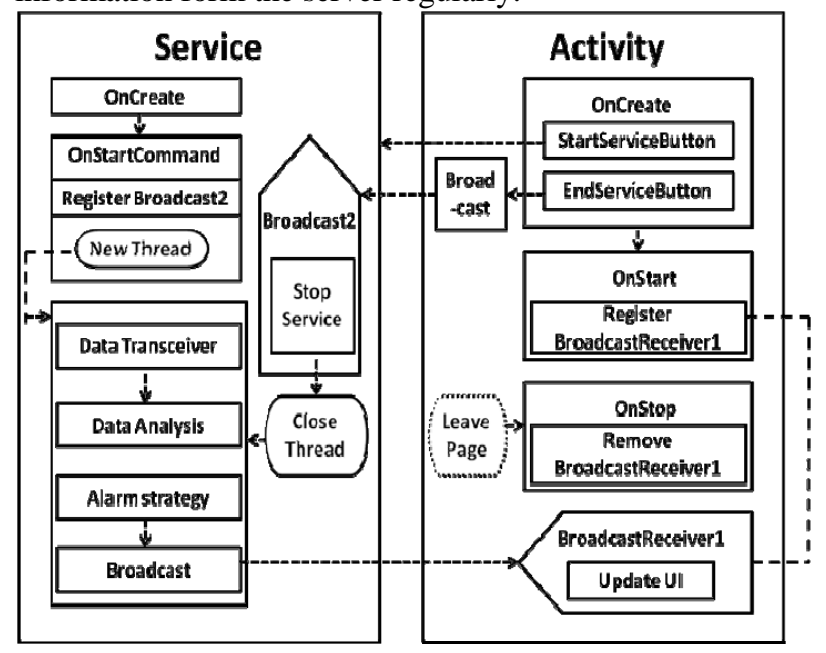

Figure 3. The Processes of Date Monitoring Service

\section{INTELLIGENT AlARM METHOD}

The environment intelligent alarm module is a key part of the method. Most of intelligent alarm method design is used currently in certain occasions in domestic, such as the fire scene alarm method which is mainly concentrated in the neural network algorithm and fuzzy system algorithm [5] [6]. The transitional environment thermal comfort evaluation model based on BP neural network proposed by Xu Hongbo etc.[7] and the Indoor environment comfort modeling and simulation method based on neural network proposed by $\mathrm{Li}$ Ting etc.[8] excellently solved the problem of the indoor Environment Monitoring. Research on environment intelligent alarm is also developing rapidly abroad. In 2009, University of Rostock, Germany studies on intelligent hospital and proposed the mobile real-time data acquisition technology in the field of preventive medicine. The method is to real-time monitoring through the physical parameters of people heart disease and their environment [9]. The University of Colorado in 2011 proposed a mobile personalized Indoor Environment Monitoring System, which has referential significance. $\mathrm{CO} 2$ as the environment 
parameter is mobile monitored. The $\mathrm{CO} 2$ concentration and the number of times of air circulation are the significant basis to define Indoor air quality standards. Finally, four levels of standards are divided to launch Intelligent Alarm [10]. Analyzing domestic and international environmental alarm method, we find that prediction method based on BP neural network is the most prevalent. In this paper, on the basis of the Indoor Air Quality Standards and Hospital Infectious Diseases Indoor Air Health Quality Requirements, we propose hospital environment intelligent alarm method based on BP neural network method in wards. Wards Environment concrete parameters are presented in Table 1.

TABLE I. WARD ENVIRONMENT PARAMETER

\begin{tabular}{|c|c|c|c|c|}
\hline Name & Parameter & Unit & Standard & Notes \\
\hline Ward & Temperature & ${ }^{0} \mathrm{C}$ & $21 \sim 24$ & \\
\cline { 2 - 5 } & Humidity & $\%$ & $30 \sim 60$ & \\
\cline { 2 - 5 } & Light & $\mathrm{lx}$ & $>=50$ & according to needs \\
\cline { 2 - 5 } & $\mathrm{CO} 2$ & $\%$ & $<=0.10$ & daily mean \\
\cline { 2 - 5 } & $\begin{array}{c}\text { Dust } \\
\text { (PM10) }\end{array}$ & $\mathrm{mg} / \mathrm{m} 3$ & $<=0.15$ & daily mean \\
\cline { 2 - 5 } & Noise & $\mathrm{dB}(\mathrm{A})$ & $<=55$ & \\
\cline { 2 - 5 } & $\ldots$ & $\ldots$ & $\ldots$ & $\ldots$ \\
\hline
\end{tabular}

For the alarm module, first of all judge whether the value of the parameter is more than the threshold value, because the instantaneous value can not be used as the alarm of the full basis, local decision judgment is necessary. After meet the requirements, then enter into the decision inference module for intelligent alarm processing. For local decision process, uses the mutative threshold algorithm:

LocalDecision $_{\mathrm{j}}=\mathrm{f}\left(\mathrm{x}_{\mathrm{j}}-\mathrm{k}^{*} \mathrm{FM}_{\mathrm{j}}\right)$

Among them, the LocalDecision $_{\mathrm{j}}$ is parameter $\mathrm{j}$ local decision results; $x_{j}$ is parameter $\mathrm{j}$ input value; $\mathrm{FM}_{\mathrm{j}}$ is parameter $\mathrm{j}$ benchmark threshold; $\mathrm{k}$ is the environment compensation correction; $\mathrm{f}(\mathrm{x})$ is the unit step function. According to different monitoring departments, in different time, each parameter value is different. When any parameters in the local decision result called LocalDecisionj appears 1 , it means the environment parameters is abnormal, and the system will immediately enter into the alarm analysis module for alarm recognition. Alarm recognition decision uses the BP neural network algorithm, as shown in Figure 4.

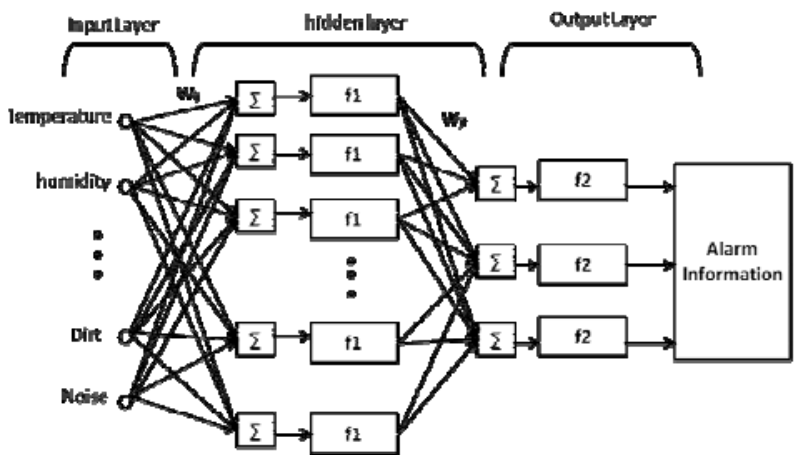

Figure 4. The Neural Network Model

Network input layer mainly includes temperature parameters, humidity parameter, light parameters, CO2 parameters, dust particles parameters and noise parameters, the parameters normalized operation is needed before the input. Hidden layer design principles is $\mathrm{n}=\sqrt{q+i}+a$, the $\mathrm{n}$ represents hidden node number; $\mathrm{q}$ is for input node number; $\mathrm{i}$ is on behalf of the output node number; a is a constant between 1 to 10 . By repeatedly collecting samples of the study, we find that the network convergence speeds is faster when the number of neurons in the network input layer is 10 and the output layer nodes are three. The output layer environmental information display as the following form: hot and humid (001), hot and dry (010), gloomy and cold (011), freezing and cold (100), noise (101), dusty (110).

The study of alarm method, according to the analysis of the research from Paris University Department of Psychology, is tied up with alarm sound. The volume, the tone and the rhythm should be various with the environment changing [11]. The system, which is mainly applied in the quiet hospital, is designed with a short alarm sound combining vibration and various rhythms. In the intelligent warning, the prompt is described as two short vibration and a gently alarm sound. However, in the intelligent alarm, the vibration becomes longer and the rhythm has an obvious change. It strengthens the level for the incident with long time hanging.

\section{RESULT}

The development environment of this method is EclipseSDK3.5.2 and ADT11.0.0, test machine is selected Samsung s5830. Testing time that mobile phone connecting to the server is less than $5 \mathrm{~s}$ through repeated test .Commanding response time between Remote server and mobile is less than 3s. Basically completed the mobile commonly access and control function. Figure 5 shows the function interface of this method, include the background monitoring service interface, the detailed department environmental information interface, the real-time environmental curve information interface.

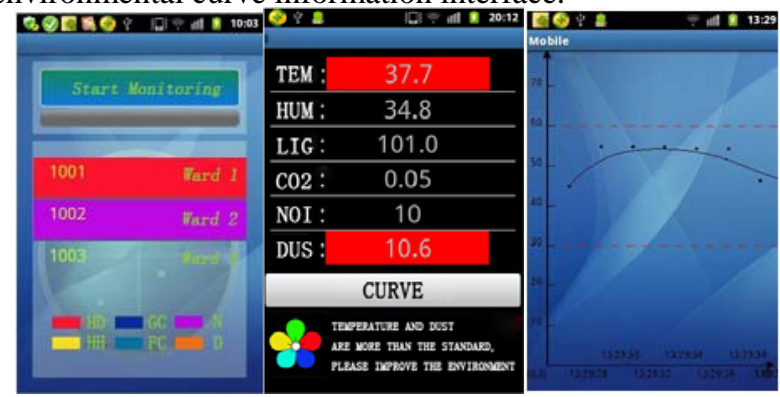

Figure 5. Main Function Interface

\section{CONCLUSION}

The intelligent hospital environment monitoring method based on the Android platform mainly adopts service, thread communication, JSON technology and so on. The data fusion method is mainly applied on the server for analysing the environment alarm information. The whole design method conforms to the national environmental monitoring 
standard. While the dust particles sensor module exists some shortcomings due to the hardware design, for example the accuracy is not high, acquisition time is a little long, which will be improved in the future. Later work mainly focuse on the analysis of the hospital environment. This intelligent hospital environment monitoring method provides an effective all-round, multi-angle solution for the hospital environmental monitoring in the future, which will make a great contribution for realizing the safe city.

\section{ACKNOWLEDGMENT}

This work was sponsored by the foundation of the Project of Informatization and Information Industry Plan of Shenyang City of China (R201201042) and the Project of College Student Innovation and Entrepreneurship Training Plan of Liaoning Province.

\section{REFERENCES}

[1] The quality standard of indoor air, The people's Republic of China National Standard, GB/T 18883-2002.

[2] Internal air quality requirements of hospital infectious disease department, Local standard of Beijing city, DB11/409-2007.

[3] Smart phones overtake client PCs in 2011. http://www.canalys.com/newsroom/smart-phones-overtake-clientpcs-2011
[4] Guanhua Wang, Improving Data Transmission in Web Applications via the Translation between XML and JSON. Communications and Mobile Computing (CMC), 2011 Third International Conference on ,2011.pp.182 - 185.

[5] Fu Jianfeng, Research of fire detection algorithm based on the data fusion technology [D], Chongqing University, 2007.

[6] Li Jian, Research of fire detection signal processing algorithms and performance evaluation methods [D], Dalian University of Technology, 2006.

[7] Xu Hongbo, Duan Mulin, Jin Quan etc. The transitional environment thermal comfort evaluation model based on BP neural network. Air conditioner of building heat ventilation ,2011,30(4):28-31.

[8] Li Ting,Chen Yuanrui. The modeling and Simulation of indoor environmental comfort neural network. Computer simulation, 2011, 28(6):189-201.

[9] Sebastian Neubert, Dagmar Arndt, Kerstin Thurow, Regina StollDr. Mobile Real-Time Data Acquisition System for Application in Preventive Medicine. ORIGINAL RESEARCH. 2010,pp.504-508.

[10] Yifei Jiang, Kun Li, Lei Tian, Ricardo Piedrahita, Xiang Yun, Omkar Mansata, Qin Lv, Robert Dick, Michael Hannigan, Li Shang. MAQS: A Personalized Mobile Sensing System for Indoor Air Quality Monitoring. In Proceedings of the 13th International Conference on Ubiquitous Computing (Ubicomp 2011). Sep. 2011.

[11] A. Guillaume, C. Drake, M. Rivenez, L. Pellieuz and V.Chastres, Perception of urgency and alarm design, in Proc. 8th Int. Conf. Auditory Display, Kyoto, Japan, 2002. 